\title{
Energy-Dependent Tunneling from Few-Electron Dynamic Quantum Dots
}

\author{
M. R. Astley, ${ }^{1,2}$ M. Kataoka, ${ }^{1}$ C. J. B. Ford, ${ }^{1}$ C. H. W. Barnes, ${ }^{1}$ D. Anderson, ${ }^{1}$ G. A. C. Jones, ${ }^{1}$ I. Farrer, ${ }^{1}$ \\ D. A. Ritchie, ${ }^{1}$ and M. Pepper ${ }^{1,2}$ \\ ${ }^{1}$ Cavendish Laboratory, University of Cambridge, J. J. Thomson Avenue, Cambridge CB3 OHE, United Kingdom \\ ${ }^{2}$ Toshiba Research Europe Limited, Cambridge Research Laboratory, \\ 260 Cambridge Science Park, Cambridge CB4 OWE, United Kingdom
}

(Received 16 May 2007; published 10 October 2007)

\begin{abstract}
We measure the electron escape rate from surface-acoustic-wave dynamic quantum dots (QDs) through a tunnel barrier. Rate equations are used to extract the tunneling rates, which change by an order of magnitude with tunnel-barrier-gate voltage. We find that the tunneling rates depend on the number of electrons in each dynamic QD because of Coulomb energy. By comparing this dependence to a saddlepoint-potential model, the addition energies of the second and third electron in each dynamic QD are estimated. The scale ( $\sim \mathrm{a}$ few $\mathrm{meV})$ is comparable to those in static QDs as expected.
\end{abstract}

PACS numbers: 73.23.Hk, 73.50.Rb, 73.63.Kv

Quantum dots (QDs) in semiconductor systems, where electrons are confined in zero-dimensional states, have been the object of much recent attention [1,2]. In a gatedefined quantum dot the number of electrons can be reduced down to one [3,4]; such single-electron QDs may form the basis of qubits in quantum computation schemes $[5,6]$. High frequency operations on QD systems have been used to observe fundamental electronic phenomena such as coherent charge oscillations [7], single- and multiple-spin dynamics [8-10], excited-state spectra [11], and elastic tunneling behavior [12], and will be necessary for quantum computation applications in semiconductor systems.

In typical QD experiments, the QDs were defined by static surface gates, and high frequency operations were achieved by applying voltage pulses to the gates. However, an alternative method has received recent attention: to use a dynamic QD defined by a surface acoustic wave (SAW) where high frequency operations are performed by moving the QD past static surface gates at a high velocity $[13,14]$. Because GaAs is piezoelectric, the strain wave of a SAW on a GaAs/AlGaAs heterostructure is accompanied by an electric potential modulation, which forms a series of oneor few-electron dynamic QDs in an empty quasi-onedimensional channel $[15,16]$. Previous experiments have attempted to observe interactions in dynamic QDs defined by SAWs [17], but to our knowledge the tunneling behavior necessary to observe complex quantum phenomena has not been seen.

In this Letter we report measurements of the nonequilibrium escape rate from one- and few-electron dynamic QDs defined by a SAW. This measurement has been carried out in static quantum dots over second [18] and millisecond [12] time scales, but the dynamic QD arrangement allows us to directly observe electron tunneling on subnanosecond time scales. The SAW-defined dynamic QDs carry electrons along the channel to a tunnel barrier, where the electrons can escape from the QD into a neighboring two-dimensional electron gas (2DEG). Observation of the tunneling current allows us to determine the tunnel rate of electrons leaving the dynamic QD, which is found to depend on the number of electrons in the dot. By fitting these rates to a simple model, we determine the addition energy of the dynamic QD. This is, to our knowledge, the first direct measurement of dynamic QD energies.

The device was made using a modulation-doped GaAs/AlGaAs heterostructure, which had a 2DEG $97 \mathrm{~nm}$ below the surface with a mobility of $160 \mathrm{~m}^{2} / \mathrm{V} \mathrm{s}$ and a carrier density of $1.8 \times 10^{15} \mathrm{~m}^{-2}$ in the dark. $\mathrm{NiCr} / \mathrm{Au}$ surface gates (shown in Fig. 1) deplete the 2DEG under negative bias to create the SAW channel and tunneling region. The SAW was generated by applying an $11.1 \mathrm{dBm}$ microwave signal from an Agilent 8648D signal generator to a transducer, made of 70 pairs of interdigitated fingers with a period of $1 \mu \mathrm{m}$, situated $2.5 \mathrm{~mm}$ from the device. Based on measurements from a device that underwent identical processing [19], we predict that this would result in a SAW amplitude of $\sim 50 \mathrm{meV}$ peak to peak. The microwave power was pulse-modulated using a Tektronix PG5110 pulse generator, with a duty ratio of $10 \mu \mathrm{s}: 500 \mu \mathrm{s}$ to minimize sample heating [19]. Measurements were carried out in a ${ }^{3} \mathrm{He}$ cryostat with a base temperature of $270 \mathrm{mK}$.

The injector gate $\left(G_{I}\right)$ is used to control the number of electrons that can enter the SAW channel. At sufficient SAW power the injected current becomes quantized to $I_{\text {in }}=N e f$, where $e$ is the electron charge and $f$ is the SAW frequency. In this regime each SAW minimum forms a dynamic QD that contains $N$ electrons, moving through the channel at the SAW velocity $\left(\sim 2800 \mathrm{~ms}^{-1}\right)$. When the dot is alongside the tunnel barrier gate $\left(G_{T}\right)$, the electrons are coupled to the reservoir and tunnel out of the dot; this tunneling process is described by $\Gamma_{n}$, the rate at which an electron leaves an $n$-electron dynamic QD. (Note that we use $N$ for the number of initially injected electrons in each $\mathrm{QD}$, whereas $n$ is the number of electrons in a QD in the tunnel barrier region). Escape of electrons from the dot 

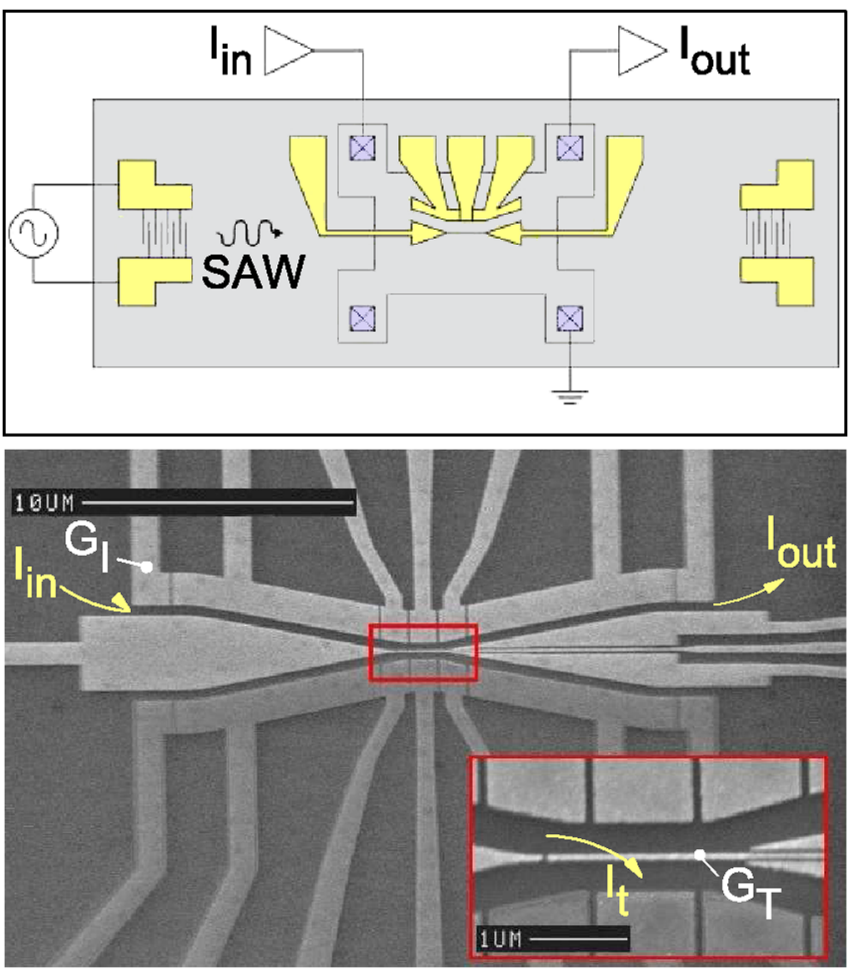

FIG. 1 (color online). Upper panel: Schematic of the device design. Lower panel: Electron micrograph of the device's surface gates. The dark shaded gates were grounded.

means the current $I_{\text {out }}$ coming out of the channel is reduced by a tunneling current $I_{t}$. The effective length of the tunnel barrier can be estimated by solving Laplace's equation for the device's surface gate voltages [20] as $\sim 1.6 \mu \mathrm{m}$, meaning the dynamic QD is coupled to the reservoir for a tunneling duration $(\tau)$ of about $600 \mathrm{ps}$. Although the exact barrier length may be smaller than this because of impurity or disorder potentials, we determine $\Gamma \tau$ in our analysis, so uncertainty in the exact value of $\tau$ does not affect our results. The remaining gates that define the channel are held at constant voltage throughout the experiment; these voltages have been carefully tuned to minimize any potential gradients in the channel, as large potential gradients could cause a loss of confinement in the dynamic QDs and lead to fluctuations from the initialized electron number $N$.

The dotted line in Fig. 2 shows $I_{\text {in }}$ as a function of the voltage applied to the injector gate. The first three quantized plateaus can be seen at multiples of $8.7 \mathrm{pA}$, which is Nef reduced by the 1:50 pulse ratio used [21]. The solid lines show $I_{\text {out }}$ for a range of voltages $\left(V_{T}\right)$ applied to the tunnel barrier - the less negative the barrier voltage, the higher the rate of tunneling out of the channel, thus the lower the value of $I_{\text {out }}$. The tunneling current $I_{t}$ is deduced from the difference between $I_{\text {in }}$ and $I_{\text {out }}$.

In previous SAW measurements, it was not possible to demonstrate that electrons were confined in a dynamic QD for the entire length of a long SAW channel (an essential feature of proposed SAW quantum circuits). An alternative

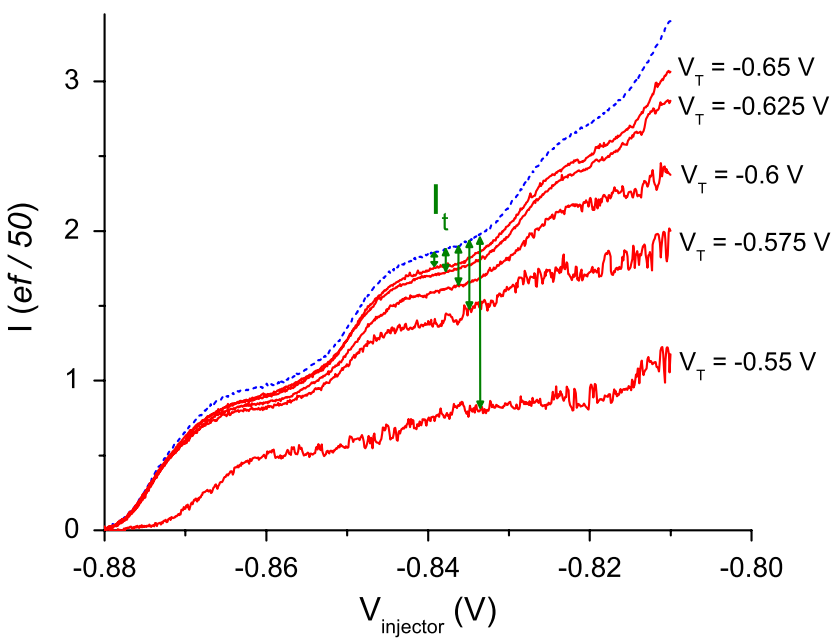

FIG. 2 (color online). $I_{\text {in }}$ (dotted line) and $I_{\text {out }}$ (solid lines) dependence on injector gate voltage for a range of barrier-gate voltages. Plateaus occur when an integer number $(N)$ of electrons occurs in each SAW minimum. $I_{t}$ is the difference between the two curves.

possibility was that quantized charge pumping occurred at a microconstriction, but subsequently electrons could escape from the dot and freely move along the channel. In our device, if electrons were not confined in dynamic QDs but were free to move in an open channel, we would expect that adding up to three electrons in a SAW cycle would not change the energy of the system. Hence such behavior would be unobservable and the ratio $I_{\text {in }} / I_{\text {out }}$ would be independent of $N$. On the other hand, if electron confinement is maintained, the energy state of the dot can vary by several meV depending on the number of electrons present and the size of the confinement potential, and thus the tunneling rate and therefore $I_{\text {in }} / I_{\text {out }}$ should be number dependent. In Fig. $3 I_{\text {in }} / I_{\text {out }}$ is shown as a function of barrier-gate voltage for $N=1,2,3$. The ratio $I_{\text {in }} / I_{\text {out }}$ is strongly dependent on $N$, indicating that the dynamic QD model correctly describes our system for at least the whole tunnel barrier region.

Control of the tunneling rate of electrons leaving a QD is needed for understanding and manipulating the quantum states within the dot. We can deduce the tunneling rate $\Gamma_{n}$ of an $n$-electron dynamic quantum dot by comparing our measurements with rate equations. Within the tunneling region, the probability $\left(P_{n}\right)$ for having $n$ electrons in the dot varies with time according to $\frac{d P_{n}}{d t}=\Gamma_{n+1} P_{n+1}-\Gamma_{n} P_{n}$ (we treat each dynamic QD as undergoing an independent tunneling event-there is a $\sim 1 \mu \mathrm{m} \sim 50 \mathrm{meV}$ barrier between electrons in neighboring dots, so there will be no wave function overlap, and the Coulomb energy of two electrons $\sim 1 \mu \mathrm{m}$ apart is only $\sim 100 \mu \mathrm{eV}$, which should have little effect). Assuming that the tunnel rates $\Gamma_{n}$ remain constant over the duration of tunneling $\tau_{n}$, that on the $I_{\text {in }}=N$ ef plateau there are exactly $N$ electrons in each SAW minimum, and that no electrons are able to 


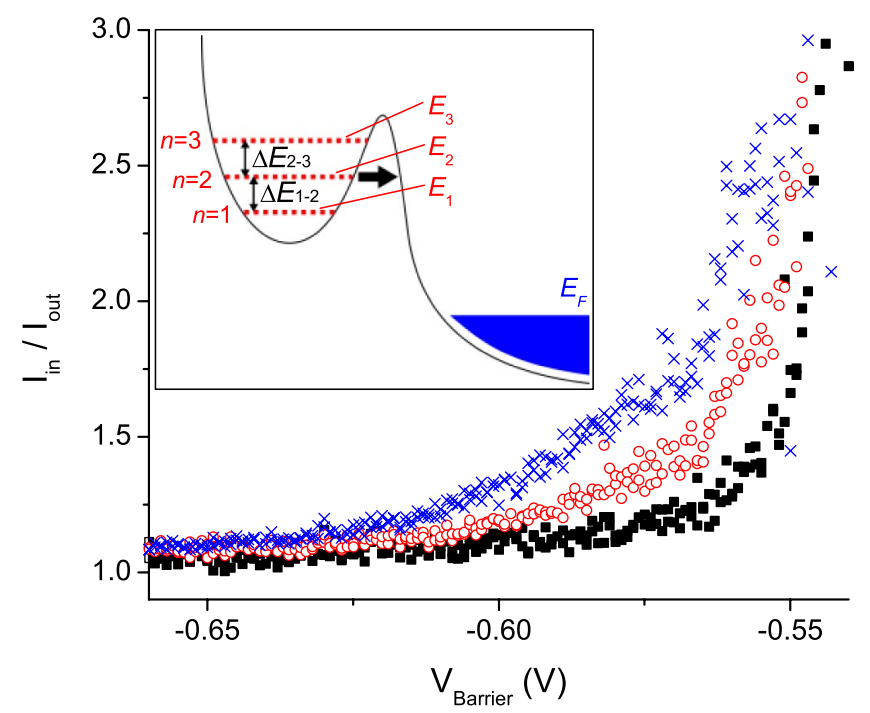

FIG. 3 (color online). The ratios $I_{\text {in }} / I_{\text {out }}$ as a function of barrier-gate voltage, taken from the $I_{\text {in }}$ plateau corresponding to $N=1(\boldsymbol{\square}), N=2(\bigcirc)$, and $N=3(\times)$. Inset: Schematic of tunneling of electrons from the dynamic QD across the barrier into the reservoir-energies of the electrons within the dot are dependent on $n$, leading to $n$-dependent tunneling rates.

tunnel back into the dot, $I_{\text {out }}=$ ef $\sum_{n=1}^{N} n P_{n}$ can be calculated as

$$
\begin{aligned}
I_{\text {out }}= & e f e^{-\Gamma_{1} \tau_{1}} \quad(N=1), \\
I_{\text {out }}= & e f\left(2 e^{-\Gamma_{2} \tau_{2}}+\frac{\Gamma_{2}}{\Gamma_{1}-\Gamma_{2}}\left(e^{-\Gamma_{2} \tau_{2}}-e^{-\Gamma_{1} \tau_{1}}\right)\right) \quad(N=2), \\
I_{\text {out }}= & e f\left[3 e^{-\Gamma_{3} \tau_{3}}+\frac{2 \Gamma_{3}}{\Gamma_{2}-\Gamma_{3}}\left(e^{-\Gamma_{3} \tau_{3}}-e^{-\Gamma_{2} \tau_{2}}\right)+\frac{\Gamma_{2} \Gamma_{3}}{\Gamma_{2}-\Gamma_{3}}\right. \\
& \times\left(\frac{1}{\Gamma_{1}-\Gamma_{3}} e^{-\Gamma_{3} \tau_{3}}-\frac{1}{\Gamma_{1}-\Gamma_{2}} e^{-\Gamma_{2} \tau_{2}}\right. \\
& \left.\left.+\frac{\Gamma_{2}-\Gamma_{3}}{\left(\Gamma_{1}-\Gamma_{2}\right)\left(\Gamma_{1}-\Gamma_{3}\right)} e^{-\Gamma_{1} \tau_{1}}\right)\right] \quad(N=3) .
\end{aligned}
$$

The assumption of exactly $N$ initial electrons is not perfect, as imperfect quantization in the SAW current leads to some dynamic QDs having $N+1$ or $N-1$ electrons [22], and there is a small possibility that electrons may be transferred between adjacent dynamic QDs after initialization. However, both of these processes should only affect a small percentage of dynamic QDs, and because $I_{\text {in }}=N$ ef there must be equal numbers of $N+1$ and $N-1$ dynamic QDs whose effects would tend to cancel each other out, so the errors caused by this assumption should be less than the measurement errors in our system. Using these equations, the values of $\Gamma_{n} \tau_{n}$ are calculated as a function of barriergate voltage in Fig. 4. The tunneling rate is varied over an order of magnitude by a single gate, which shows great promise for making future SAW quantum devices.

The data in Fig. 4 are fitted using the analytical solution for the transmission probability of noninteracting electrons

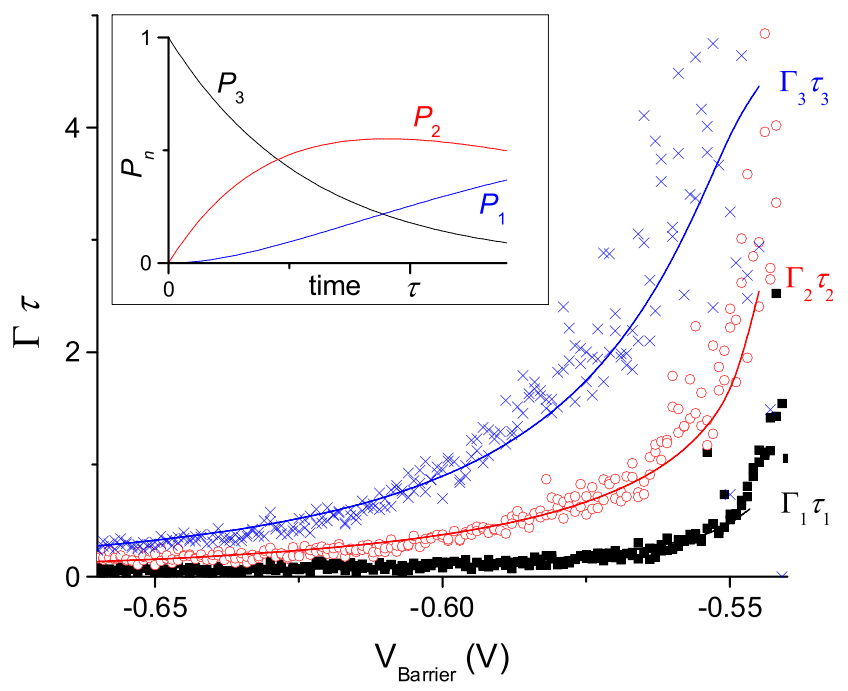

FIG. 4 (color online). Dependence of the calculated tunneling rates $\Gamma_{n}$ on barrier-gate voltage for one $(\boldsymbol{\square})$, two $(\bigcirc)$, and three $(\times)$ electrons in the dynamic $\mathrm{QD}$, normalized by the tunneling time $\tau_{n}$. The solid lines show fits based on the tunneling probability of noninteracting electrons incident on a saddle-point potential, as described in the text. Inset: Example of the time evolution of $P_{n}$ for $3 e f$ injection, using tunnel rates for $V_{T}=$ $-0.575 \mathrm{~V}$.

through the saddle-point potential $V(x, y)=V_{0}-$ $\frac{1}{2} m^{*} \omega_{x}^{2}+\frac{1}{2} m^{*} \omega_{y}^{2}[23,24]$ :

$$
\begin{aligned}
T_{i, j} & =\delta_{i, j} \frac{1}{1+e^{-\pi \epsilon}}, \\
\text { where } \epsilon & =\frac{2\left[E_{n}-\hbar \omega_{y}\left(i+\frac{1}{2}\right)-V_{0}\right]}{\hbar \omega_{x}},
\end{aligned}
$$

$V_{0}$ is the potential at the center of the barrier, $m^{*}$ is the effective mass of the electron, $\omega_{x}\left(\omega_{y}\right)$ controls the curvature of the barrier perpendicular (parallel) to the barrier, $\delta_{i, j}$ is the Kronecker delta function, $E_{n}$ is the energy of the incident electron, and, assuming the electron tunnels through the one-dimensional ground state, the subband index $i=0$. The transmission probabilities are converted to tunneling probabilities by multiplying by a free parameter which describes the number of attempts the electron makes at tunneling in the time $\tau$, and the other terms in the expression can be related to changes in the tunnel barrier voltage $\left(V_{T}\right)$ by assuming a simple capacitor model: $\Delta V_{0}=\alpha_{V_{0}} \Delta V_{T}$ and $\frac{1}{2} m^{*} \Delta \omega_{x}^{2}=\alpha_{\omega_{x}} \Delta V_{T}$, where each $\alpha$ is a constant relating the coupling of the gate to the barrier potential; $\omega_{y}$ is determined by the SAW potential amplitude and so remains constant. We estimate $\alpha_{V_{0}}=0.62 \pm$ 0.01 by applying a bias potential to the 2DEG until a breakdown current starts to flow through the upper channel, which is expected to occur when the Fermi energy of the 2DEG is level with the top of the barrier. From the fitting parameters in Table I, we can extract the addition energies $\Delta E_{n \rightarrow n+1}$ for an $n$ electron dynamic QD. We find 
TABLE I. Fitting parameters from Fig. 4, used to derive the addition energies of the dynamic QD.

\begin{tabular}{lccc}
\hline \hline & $\frac{2 E_{n}-\hbar \omega_{y}}{\hbar \sqrt{2 \alpha_{\omega_{x}} / m^{*}}}$ & $\frac{2 \alpha_{V_{0}}}{\hbar \sqrt{2 \alpha_{\omega_{x}} / m^{*}}}$ & $E_{n}-\frac{1}{2} \hbar \omega_{y}$ \\
& $\left(\mathrm{~V}^{1 / 2}\right)$ & $\left(\mathrm{V}^{-1 / 2}\right)$ & $(\mathrm{meV})$ \\
\hline$n=1$ & $0.0013 \pm 0.0004$ & & $0.27 \pm 0.08$ \\
$n=2$ & $0.015 \pm 0.002$ & $3.050 \pm 0.017$ & $2.9 \pm 0.4$ \\
$n=3$ & $0.084 \pm 0.006$ & & $17.0 \pm 1.2$ \\
\hline \hline
\end{tabular}

$\Delta E_{1 \rightarrow 2}=2.6 \pm 0.4 \mathrm{meV}$ and $\Delta E_{2 \rightarrow 3}=14.1 \pm 1.3 \mathrm{meV}$ (these errors are from the fitting; there may be other errors caused by the assumptions in the model that have not been accounted for).

The energy of the dynamic QD will be increased by a Coulomb repulsion when adding an electron to the dot. The constant-interaction model of a QD predicts $\Delta E_{n \rightarrow n+1}=$ $e^{2} / 2 C+\delta E_{\mathrm{sp}}$, with a capacitance $C$, at equal gate voltages, and where $\delta E_{\mathrm{sp}}$ is the single-particle energy spacing (for a discussion of Coulomb energies within QDs, including the limitations of this constant-interaction model, see Ref. [2]). This predicts the ratio $\Delta E_{2 \rightarrow 3} / \Delta E_{1 \rightarrow 2} \approx 1$, whereas we find $\Delta E_{2 \rightarrow 3} / \Delta E_{1 \rightarrow 2}=5.4 \pm 1.0$. The difference is too large to be attributed solely to the singleparticle energy - we suggest that the large variation in addition energies may be due to the complexities of the exchange and Coulomb interactions in few-electron QDs, which would require a self-consistent theory of electronelectron interactions to model accurately (note that the distance from QD to reservoir 2DEG is greater in our dynamic QDs than in previous static QD measurements, which will reduce the screening of the Coulomb interaction by the reservoir and could result in larger electron-electron effects). However, the very large discrepancy may also suggest that assumptions in the saddle-point tunneling model (e.g., ignoring electron-electron interactions in the tunneling process or assuming the rate is only sensitive to the potential at the tunnel barrier) are affecting the calculation, but while our measured addition energies may contain inaccuracies due to the approximations incorporated into our model, we note that the energies are of comparable order of magnitude to those measured in static few-electron quantum dots [3,4].

In summary, we have demonstrated observations of tunneling on a $\sim 600$ ps time scale by confining electrons in dynamic QDs using a SAW. Tunnel rates may be determined from the currents flowing through the device by using rate equations. The tunnel rates are dependent on the barrier voltage applied and on the number of electrons in the dot; fitting these dependencies to a saddle-point tunneling model gives addition energies that we attribute to the Coulomb interaction. The physical behavior of electrons confined to dynamic QDs is found to be similar to that of electrons in static QDs, indicating that dynamic QDs can provide an additional method of probing the fundamental behavior of electrons in QDs.

This work was part of the QIP IRC (Grant No. GR/ S82176/01). M. R. A. thanks Toshiba Research Europe Ltd. and UK EPSRC for funding. Calculations of device potentials were carried out using the GatesCalc program written by A. L. Thorn.

[1] L. P. Kouwenhoven and C. M. Marcus, Phys. World 11, 35 (1998).

[2] L. P. Kouwenhoven, D. G. Austing, and S. Tarucha, Rep. Prog. Phys. 64, 701 (2001).

[3] S. Tarucha et al., Phys. Rev. Lett. 77, 3613 (1996).

[4] M. Ciorga et al., Phys. Rev. B 61, R16315 (2000).

[5] D. Loss and D. P. DiVincenzo, Phys. Rev. A 57, 120 (1998).

[6] L. M. K. Vandersypen et al., in Quantum Computing and Quantum Bits in Mesoscopic Systems, edited by A. J. Legget, B. Ruggiero, and P. Silvestrini (Kluwer Academic/ Plenum, New York, 2003), p. 201; arXiv:quant-ph/ 0207059.

[7] T. Hayashi et al., Phys. Rev. Lett. 91, 226804 (2003).

[8] J. M. Elzerman et al., Nature (London) 430, 431 (2004).

[9] J. R. Petta et al., Science 309, 2180 (2005).

[10] F. H. L. Koppens et al., Nature (London) 442, 766 (2006).

[11] J. M. Elzerman et al., Appl. Phys. Lett. 84, 4617 (2004).

[12] K. MacLean et al., Phys. Rev. Lett. 98, 036802 (2007).

[13] C. H. W. Barnes, J. M. Shilton, and A. M. Robinson, Phys. Rev. B 62, 8410 (2000).

[14] R. Rodriquez et al., Phys. Rev. B 72, 085329 (2005).

[15] J. M. Shilton et al., J. Phys. Condens. Matter 8, L531 (1996).

[16] V. I. Talyanskii et al., Phys. Rev. B 56, 15180 (1997).

[17] M. Kataoka et al., Physica E (Amsterdam) 34, 546 (2006).

[18] J. Cooper et al., Physica E (Amsterdam) 6, 457 (2000).

[19] R. J. Schneble et al., Appl. Phys. Lett. 89, 122104 (2006).

[20] J. H. Davies, I. A. Larkin, and E. V. Sukhorukov, J. Appl. Phys. 77, 4504 (1995).

[21] M. Kataoka et al., J. Appl. Phys. 100, 063710 (2006).

[22] A. M. Robinson and V. I. Talyanskii, Phys. Rev. Lett. 95, 247202 (2005).

[23] M. Büttiker, Phys. Rev. B 41, R7906 (1990).

[24] J. N. L. Connor, Mol. Phys. 15, 37 (1968). 